\title{
Speaking up against unsafe unprofessional behaviours: the difficulty in knowing when and how
}

\author{
Brian M Wong, ${ }^{1}$ Shiphra Ginsburg ${ }^{2}$
}

'Medicine, Sunnybrook Health Sciences Centre, University of Toronto, Toronto, Ontario, Canada

${ }^{2}$ Medicine, Mount Sinai Hospital and Wilson Centre for Research in Education, University of Toronto, Toronto, Ontario, Canada

\section{Correspondence to} Dr Shiphra Ginsburg, Department of Medicine, University of Toronto, M5G1X5 433-600 University Ave, Toronto, Ontario M5G1X5, Canada; shiphra.ginsburg@utoronto.ca

Accepted 7 June 2017 Published Online First 22 July 2017

\section{Linked}

- http://dx.doi.org/10.1136/ bmjas-2016-006284

\section{CrossMark}

To cite: Wong BM, Ginsburg S. BMJ Qual Saf 2017;26:859-862.
Patient safety is a core focus now in medical education, with an increasing number of training programmes educating learners about its key tenets. ${ }^{1-3}$ Residents now undergo formal training about the importance of contributing to a culture of safety by speaking up to avoid errors or harm, but still face difficulties enacting these behaviours in practice. ${ }^{4}$ In this issue of the journal, Martinez et al have attempted to tease out differences in speaking-out behaviours between traditional and professionalism-related patient safety threats. ${ }^{5}$ The authors have raised several interesting points worthy of further exploration. In this editorial we focus on unprofessional behaviour as a potential threat to patient safety and propose some new ways of thinking about how to integrate research and lessons from both the patient safety and professionalism literature.

In the study by Martinez and colleagues, residents reported witnessing unprofessional behaviour more frequently than they observed patient safety breaches $(75 \%$ vs $46 \% ; \mathrm{p}<0.001) .{ }^{5}$ They also reported speaking up less frequently about unprofessional behaviour than about traditional safety threats $(46 \%$ vs $71 \% ; \mathrm{p}<0.001)$ despite their view that this is important for patient safety. When participants were faced with two safety scenarios (one meant to represent a traditional threat to patient safety and one focused on unprofessional behaviour), the same pattern was seen. The authors suggest that professionalism-related safety threats are more difficult to speak up about because, among other things, they are perceived to be more deliberate and more likely due to character flaws and thus are less likely to change even if reported. ${ }^{67}$ Residents were also concerned about alienation from the team and potential for retaliation. The authors focus on two instruments that assess the 'culture' for safety and reporting, and conclude that measuring the environment could draw our attention to units or teams that are most in need of intervention.

While intriguing, this study has several important caveats to consider. First, the study relies on self-reports from residents, both for the reports of observed behaviours as well as regarding their likelihood of speaking up. What residents perceive as unprofessional may be quite variable depending on the context, the definition of professionalism and the framing of the question. Second, the authors' use of only two scenarios is problematic for several reasons that may limit reproducibility. The differences in willingness to speak up may not be primarily related to one scenario having a 'professionalism' issue - there is also a different sense of immediacy: breaching a sterile field is directly linked to the risk of infection while the potential effect of a resident not paying attention on rounds may not have any consequence at all (the resident may not have responsibility for looking after those patients) or not until much later (there may be other opportunities to bring them up to speed if they do need to assume care). Thus the urgency of speaking up in the moment may be one reason for the difference in responses. That said, we do think the findings are supported by the literature illustrating the difficulties of speaking up in the face of unprofessional behaviour, but should likely be viewed as hypothesis-generating (to be validated with multiple different types of professionalism issues) rather than conclusive.

One important concept the authors draw our attention to is that perhaps professionalism has not fully been 
acknowledged as being linked to patient safety in the first place. While in the past it may have been possible to separate out professionalism as being 'soft' and not necessarily essential to safe patient care, emerging evidence illustrates that this view can no longer be supported. For example, one recent multicentre study reported a correlation between the frequency of patient (or family member) observations of concerning professional behaviours in their physicians and worse medical and surgical outcomes. ${ }^{8}$ Even in a simulated setting, researchers have reported that being exposed to rude behaviour resulted in diminished team performance, including a decrease in diagnostic and therapeutic measures. ${ }^{9}$ It is possible that aggressive behaviours such as rudeness or intimidation are especially linked to poorer outcomes. However, these findings have also been seen with poor 'relational coordination' ${ }^{10}$ among teams, which includes communication and mutual respect - elements present in the 'unprofessional' scenario in Martinez et al's paper. There is also abundant evidence demonstrating the onerous difficulty of speaking up when professionalism is at stake, whether participants are learners, ${ }^{11}$ peers ${ }^{12}$ or even faculty. ${ }^{13}$ The current article adds to this body of evidence and urges us to address the environment in order to design appropriate interventions.

In order to address these concerns, we will need a multipronged approach. First, there is a need to create broad awareness regarding the link between patient safety and professionalism concerns, and acknowledgement that providers have a responsibility to mitigate harms associated with unprofessional behaviours. One explanation for why residents in this study might have felt more empowered to speak up in the setting of patient safety breaches as opposed to professionalism breaches could relate to the way patient safety has been positioned in healthcare over the past 15 years. The overarching sentiment that medical errors relate primarily to 'systems' issues or that we should avoid 'blame and shame' naturally drew attention away from the 'individual' or the 'person-related' contributions to patient safety incidents. While 15 years ago there was a clear need to err towards emphasising the role of the system (to overcome an existing culture that used to point fingers at individuals when bad things happened), it is possible that the pendulum shifted too far in the direction of always 'blaming' the system. ${ }^{14}$ And so when faced with the prospect of speaking out against unprofessional behaviour, it is not entirely surprising that this might run counter to what has been emphasised in relation to promoting a strong safety culture.

However, the field of patient safety is now shifting away from a 'no-blame' culture and towards promoting a 'just culture', 'which differentiates blameworthy from blameless acts'. ${ }^{14} \mathrm{~A}$ just culture highlights the need to address both system deficiencies as well as manage individual behavioural choices. ${ }^{15}$ It acknowledges that individual practitioners should not be held responsible for mistakes or errors that they commit in a system that is poorly or suboptimally designed; however, individuals are still very much responsible for their choices and actions regardless of the shortcomings in a system. Some actions can be considered 'at-risk' behaviours, such as taking short cuts or not completing important clinical tasks in a timely manner (eg, late signing off on discharge summaries), or perhaps, as in the example in Martinez et $a l,{ }^{5}$ not paying attention at rounds. Other behaviours can be considered reckless, such as deliberately ignoring key safety steps, which puts patients at risk. The latter 'reckless behaviour' category would encompass what we normally think of as severe professionalism issues (eg, the 'disruptive physician', or falsifying patient care records), and provides a lens to view how professionalism concerns can link to patient safety, as well as guide the optimal way to respond to such instances. If nothing else, it can begin to normalise thinking around the important contribution that individual unprofessional behaviours can play in putting patients at risk.

But even with heightened awareness of the link between professionalism and patient safety, there remains the issue of how best to respond when such an incident occurs. Like any other intervention, we would not favour a 'one-size fits all' approach — in other words, it should not be assumed just because one observes unprofessional behaviour that speaking up in the moment is the best way to address it. Instead, we propose one possible framework that could provide guidance as to the optimal action to take when one encounters an unsafe unprofessional behaviour (box 1).

Once it has been decided that a particular unprofessional behaviour could put patient safety at risk, there are likely a variety of contextual factors that influence whether speaking up in the moment is appropriate and warranted. We believe that the most important consideration relates to the immediacy of the unprofessional behaviour and the likelihood that it puts patient safety at risk. For example, a situation where a surgeon arrives to work intoxicated is much more concerning in the moment than a surgeon who is rude to other interprofessional team members in the operating theatre. Both examples have the potential

Box 1 Framework to guide speaking-up action in the setting of unsafe, unprofessional behaviours

Is this an example of an unprofessional behaviour that could put patient safety at risk?

- Is there an immediacy to the situation where speaking up in the moment is the optimal approach to mitigate harm or uphold patient safety?

- What communication approach should be used to communicate concern? 
to result in harm, but speaking up and interrupting the first scenario of an intoxicated colleague is much more urgent given the direct and immediate harm that could come to the patient. These examples are fairly clear, yet we acknowledge that in many instances it might not be possible to know all of the downstream effects that may occur as a result of a behaviour nor how immediate a risk a given behaviour may represent. For example, instead of being rude to the other interprofessional team members, what would the appropriate response be if the surgeon threw a surgical instrument onto the tray in apparent frustration? While this likely poses no immediate threat to the patient, it could be harmful to a team member, yet if the surgeon is already frustrated because the case is not going well, then speaking up in the moment may cause a distraction and could ultimately do more harm than good. Recognising all the contextual factors that influence the immediacy of the concern is critical in deciding whether speaking up in the moment is needed.

Once it has been determined that speaking up is needed, we need to equip front-line providers with structured approaches to voicing their concerns. Graded assertiveness frameworks exist to support communication and team training in patient safety (ie, TeamSTEPPS ${ }^{16}$ promotes the use of CUS - 'concern', 'uncertain', 'safety' ${ }^{17}$; and Healthcare Performance Improvement ${ }^{18}$ promotes the use of ARCC - 'ask', 'request', 'concern', 'chain of command'), and these could provide useful guidance as examples for how unprofessional behaviours might be addressed in the moment. Teaching residents how to apply such communication approaches and practising their use is one concrete way to encourage the use of desired speaking-up behaviours when appropriate. Equally important is helping residents understand how to balance the need to interrupt the behaviour urgently and the risk of personal embarrassment to the individual. What is needed is a set of guiding principles to help residents appreciate when it is necessary to interrupt workflow and raise concern immediately (and potentially in front of other colleagues and trainees) versus waiting and taking the first available opportunity privately to voice their concern regarding the unprofessional behaviour.

Of course, we are not naïve to the fact that awareness and ability are necessary but not sufficient. One major barrier, especially in teaching environments, is the personal, professional and academic risk to those who speak up to halt unprofessional behaviours. ${ }^{19}$ In one simulation study anaesthesia residents were unwilling to speak up to an attending physician who was knowingly ordering blood for a bleeding Jehovah's Witness patient, fearing potential consequences related to their future academic standing. ${ }^{20}$ Unwillingness to give honest feedback to attending physicians may also increase with higher levels of training. ${ }^{21}$
What is the way forward? Undoubtedly, there is a clear role for curriculum change, and perhaps even more importantly, faculty development to ensure that appropriate role modelling takes place in the clinical learning environment to support speaking-up behaviours to arrest both professionalism and patient safety concerns that put patients at risk. There is likely a role for regulation as well; for example, the Accreditation Council for Graduate Medical Education (ACGME) already lists in its Clinical Learning Environment Review programme six key areas, two of which are patient safety and professionalism. ${ }^{22}$ This may represent a nice avenue to garner greater awareness about the overlap between patient safety and professionalism. Finally, we believe that there is much that can be gained from building bridges between the scholarly fields of patient safety and professionalism, as we have tried to model in writing this paper one of us (SG) is fully steeped in the world of professionalism, while the other (BMW) has been actively involved in advancing patient safety. We have much to learn from each other, and together we can jointly focus on our shared goals of improving professional culture to ensure high-quality patient care.

Contributors Both authors contributed equally to the conception, writing and editing of the manuscript.

Competing interests None declared.

Provenance and peer review Commissioned; internally peer reviewed.

(C) Article author(s) (or their employer(s) unless otherwise stated in the text of the article) 2017. All rights reserved. No commercial use is permitted unless otherwise expressly granted.

\section{REFERENCES}

1 Wong BM, Etchells EE, Kuper A, et al. Teaching quality improvement and patient safety to trainees: a systematic review. Acad Med 2010;85:1425-39.

2 Nasca TJ, Weiss KB, Bagian JP. Improving clinical learning environments for tomorrow's physicians. N Engl J Med 2014;370:991-3.

3 Jones AC, Shipman SA, Ogrinc G. Republished: Key characteristics of successful quality improvement curricula in physician education: a realist review. Postgrad Med J 2015;91:102-13.

4 Weiss KB, Bagian JP. CLER Evaluation Committee. Challenges and Opportunities in the Six Focus Areas: CLER National Report of Findings 2016. J Grad Med Educ 2016;8(2 Suppl 1):25-34.

5 Martinez W, Lehmann LS, Thomas EJ, et al. Speaking up about traditional and professionalism-related patient safety threats: a national survey of interns and residents. BMJ Qual Saf 2017;26:869-80.

6 Elkin K, Spittal MJ, Elkin D, et al. Removal of doctors from practice for professional misconduct in Australia and New Zealand. BMJ Qual Saf 2012;21:1027-33.

7 Wachter RM. Disciplining doctors for misconduct: character matters, but so does competence. BMJ Qual Saf 2012;21:976-8. 
8 Cooper WO, Guillamondegui O, Hines OJ, et al. Use of unsolicited patient observations to identify Surgeons with increased risk for postoperative complications. JAMA Surg 2017;152:522-8.

9 Riskin A, Erez A, Foulk TA, et al. Rudeness and Medical Team Performance. Pediatrics 2017;139:e20162305.

10 Gittell JH, Bierbaum B, et al. Impact of relational coordination on quality of care, postoperative pain and functioning, and length of stay: a nine-hospital study of surgical patients impact of relational coordination on quality of care, postoperative pain and functioning, and length of stay a nine-hospital study of surgical patients. Med Care 2000;38:807-19.

11 Sydor DT, Bould MD, Naik VN, et al. Challenging authority during a life-threatening crisis: the effect of operating theatre hierarchy. Br J Anaesth 2013;110:463-71.

12 Arnold L, Shue CK, Kritt B, et al. Medical students' views on peer assessment of professionalism. J Gen Intern Med 2005;20:819-24.

13 Bryden P, Ginsburg S, Kurabi B, et al. Professing professionalism: are we our own worst enemy? Faculty members' experiences of teaching and evaluating professionalism in medical education at one school. Acad Med 2010;85:1025-34.
14 Wachter RM, Pronovost PJ. Balancing "no blame" with accountability in patient safety. $N$ Engl J Med 2009;361:1401-6.

15 Dekker S. Just culture: balancing Safety and Accountability. 2nd ed. Surrey, UK: Ashgate Publishing Ltd;, 2012.

16 Agency for Healthcare Research and Quality. TeamSTEPPS Fundamentals Course: Module 6. Mutual Support. Rockville, USA: Agency for Healthcare Research and Quality, 2014.

17 Simons SL. Using CUS words in the NICU. Neonatal Netw 2008;27:423-4.

18 Graham E. In Pursuit of High Reliability. Press Ganey Publication, 2016.

19 Lucey C, Levinson W, Ginsburg S, et al. Medical Student Mistreatment. JAMA 2016;316:2263-4.

20 Bould MD, Sutherland S, Sydor DT, et al. Residents' reluctance to challenge negative hierarchy in the operating room: a qualitative study. Can J Anaesth 2015;62:576-86.

21 Willett RM, Lawson SR, Gary JS, et al. Medical student evaluation of faculty in student-preceptor pairs. Acad Med 2007;82:S30-S33.

22 Weiss KB, Bagian JP, Wagner R. CLER pathways to excellence: expectations for an optimal clinical learning environment (Executive Summary). J Grad Med Educ 2014;6:610-1. 